Alzate, N.; Santos-Boada, G.; De Almeida, J. Decision making under uncertainty for the deployment of future networks in IoT scenarios. A: International Conference on Broadband Communications, Networks, and Systems. "Broadband Communications, Networks, and Systems, 11th EAI International Conference, BROADNETS 2020: Qingdao, China, December 11-12, 2020: proceedings". Berlín: Springer, 2020, p. 174-184. ISBN 978-3-030-68737-3.

The final authenticated version is available online at https://doi.org/10.1007/978-3-030-68737-3

\title{
Decision Making under Uncertainty for the deployment of future networks in IoT Scenarios*
}

\author{
Néstor Alzate Mejía ${ }^{1,2}[0000-0003-3287-643 X]$, Germán Santos \\ Boada $^{2[0000-0002-9528-2287]}$, and José Roberto de Almeida \\ Amazonas $2,3[0000-0001-9589-0373]$ \\ 1 Cooperative University of Colombia, Santiago de Cali, Colombia \\ 2 Universitat Politècnica de Catalunya, Barcelona, Spain \\ 3 Escola Politécnica of the University of São Paulo, SP, Brazil \\ nestor.alzate@upc.edu, \{german, amazonas\}@ac.upc.edu
}

\begin{abstract}
The main characteristic of various emerging communication network paradigms in the dimensioning, control and deployment of future networks is the fact that they are human-centric, entailing closelyknit interactions between telematics and human activities. Considering the effect of user behavior, whose dynamics are difficult to model, new uncertainties are introduced in these systems, bringing about network resource management challenges. Within this context, this study seeks to review different decision-making computational methods in conditions of uncertainty for Internet of Things scenarios such as smart spaces, and industry 4.0, through a systematic literature review. According to our research results, a new paradigm for computationally capturing and modeling human behavior context must be developed with the purpose of improving resource management.
\end{abstract}

Keywords: Uncertainty · Resource Management · Decision Making.

\section{Introduction}

Different approaches to Data Communications Networks (DCN) have evolved gradually in tandem with technological advances. However, these changes entail new network resource management challenges, which must be overcome to meet the objectives from emerging approaches.

Consequently, in relation to traditional DCN management, performance is examined with regards to quantitative technical data, such as package delivery delays, the number of packages that were not delivered to their destination, transmission speeds between origins and destinations, among others. Based on

\footnotetext{
* This work has been partially supported by the Spanish Ministry of Economy and Competitiveness under contract TEC2017-90034-C2-1-R (ALLIANCE project) that receives funding from FEDER. Moreover, it has been partially supported by the Spanish Thematic Network under contract RED2018-102585-T (Go2Edge) and by the aid granted by the Sinfoni project INV2733 of the Cooperative University of Colombia.
} 
this focus, data is provided by electronic devices such as sensors, and mobile and network equipment.

Further, another burgeoning DCN-related concept is a human-centric network $(\mathrm{HCN})$, which proposes the optimization of both services and network applications by centering their decisions on the wellbeing of individuals. To accomplish this, network performance is assessed by considering both traditional quantitative and qualitative data. Qualitative data generated by individuals, either directly through opinions posted on web pages or social media or through interactions between individuals and their devices, which log human activities on several platforms.

This growing and closely knit interaction between individuals and their online activity-tracking devices has changed the roles played by individuals from passive to active in terms of DCNs [9], [11]. In this new role, human activities determine network usage behaviors. Therefore, the uncertainty produced by individuals through their perceived experiences influences DCN performance to a greater extent.

Diverse studies have approached these novel phenomena from a wide range of perspectives. For example, [12] studied diverse machine-learning techniques that may be implemented to support decision making and knowledge extraction, grounded on feedback from Internet of Things (IoT) paradigms and cyberphysical systems when combined through cloud and fog computing. Within the Industry 4.0 context, [22] developed a system implementation architecture that interacts between human agents and machines in the manufacturing sector. This proposed method included five levels with different types of challenges to overcome. With regards to smart spaces, in their work, [7] proposed an online framework, rooted on a logical Markovian network, for developing voice-driven home automation systems aimed at improving comfort and autonomy at home, thus addressing uncertainty based on context awareness. In their study on SelfOrganized Networks, [16] discussed a decision-making framework supported by various machine-learning algorithms that could manage fifth-generation mobile network resources. Their novel approach was to use software-defined networks (SDN) and network function virtualization (NFV) as decision-making technologies. However, they do not consider qualitative data.

Therefore, $\mathrm{HCN}$ features can be used in application domains for concepts such as IoT, Industry 4.0 and smart spaces. For these concepts, inherent interactions between individuals and machines suggest that computational methods can be combined with other disciplines to improve the modeling of this phenomenon.

\section{Computational Methods for Decision Making Under Uncertainty}

Decision making involves selecting of the best option from a specific set to solve a given problem in the best possible manner. An agent is an entity that makes decisions according to its interaction with the environment. Agents can be persons, robots, and even entities implemented through software [19]. This research 
study centers on computational decision-making systems, especially those that accept designs with a significant degree of process automation and that are able to handle uncertainty. A classification of the most significant methods described in the literature is listed in Fig. 1.

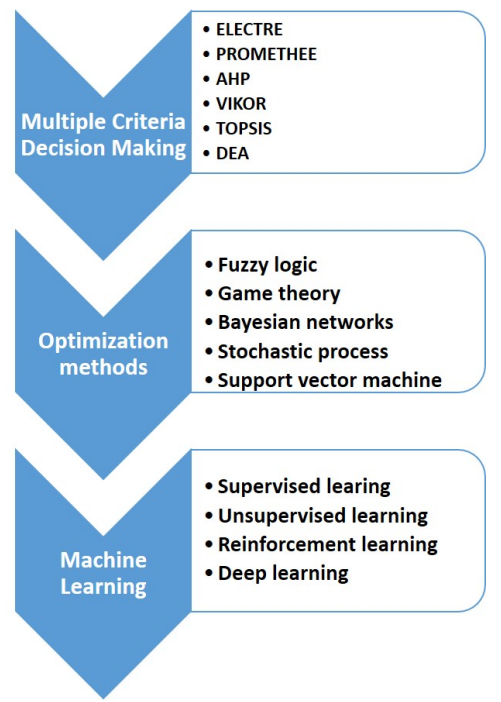

Fig. 1. Classification of Computational Methods for Decision Making.

\subsection{Multiple Criteria Decision Making}

In decision making, a set of alternatives is usually assessed by various criteria, with different weights according to their relevance to the issue at hand [4]. The problem lies in how to assign appropriate weights to each criterion. To address this situation, several Multiple Criteria Decision-Making (MCDM) tools have been extensively studied [2], [13]. Therefore, there are different MCDM methods which may be classified depending on the academic currents from the European, American, and other schools.

The European school uses the following methods:

- Elimination and Choice Expressing Reality (ELECTRE): it is used to eliminate non-viable solution alternatives. This method is usually combined with another MCDM method to minimize execution times, as in the following studies: [28], [35], [10].

- Preference Ranking Organization Method for Enrichment of Evaluations (PROMETHEE): this method is founded on mathematical and psychological methods used to develop an external classification for various alternatives, thus helping agents to choose the option that best serves its purpose as per 
its own understanding of the issue. This method has been assessed in several fields, such as for infrastructure construction [15], the electric power sector [32], [1], and engineering decision making [29], among others.

The North American school proposes the following method:

- Analytic Hierarchy Process (AHP): this method is structured to manage complex decisions based on appraisals and assessments, combining physical and psychological elements which are usually conflicting. In studies such as [25], this is evident because decision making is tackled under uncertainty or based on subjective product recommendations from consumers [21].

From different origins we can name:

- Multicriteria Optimization and Compromise Solution (VIKOR): is a method for determining the best possible solution, especially when dealing with conflicting options or with different units of measurement. In fact, there are several proposals for this method. For example, one is to combine this method with other techniques [27] to develop a method for assessing feelings in social media, for group decision-making processes [24] or for evaluating airline service quality [26].

- Technique for Order of Preference by Similarity to Ideal Solution (TOPSIS): this method seeks to find an alternative solution with the shortest and longest Euclidean distance from the optimum positive solution and the optimum negative solution, respectively. These solutions are mostly addressed in studies where this method is supplemented by additional algorithms, as shown in [33], [8], [5].

- Data Envelopment Analysis (DEA): it is a methodology created to assess the relative efficiencies of comparable entity sets by solving a series of mathematical programming models [18].

\section{Internet of Things Application Domain}

Internet of Things is a paradigm that arises from the possibilities foreseen by the communications technologies that emerged in the 1990s, such as RadioFrequency Identification (RFID). Kevin Ashton initially introduced the term IoT at a presentation in 1999 [3]. From then onwards, organizations such as the International Telecommunications Union (ITU), the Institute of Electrical and Electronic Engineers (IEEE), the European Telecommunications Standards Institute (ETSI), and the Internet Engineering Task Force (IETF), among others, have worked on its standardization. However, they have not reached a unique definition for IoT yet. In our view, an accurate definition is provided by an EUfunded project known as Coordination and Support Action for Global RFIDRelated Activities and Standardization (CASAGRAS), which defines it as "A global network infrastructure, linking physical and virtual objects through the exploitation of data capture and communication capabilities. This infrastructure includes existing and evolving Internet and network developments. It will offer 
specific object-identification, sensor and connection capability as the basis for the development of independent cooperative services and applications. These will be characterized by a high degree of autonomous data capture, event transfer, network connectivity, and interoperability" [17].

\subsection{Mobile Wireless Sensor Network}

A Mobile Wireless Sensor Network (MWSN) evidences a case of uncertainty within an IoT network, as shown in Fig. 2. The MWSN resources are limited, especially in terms of processing capacity and power independence. In this scenario, multiple mobile wireless sensors installed on drones intercommunicate without requiring the infrastructure of a physical network to monitor and identify intruders in specific neighborhood areas. Fig. 2 (A) provides a late-night example, at hours when it is unlikely that people will be out in the streets. Nevertheless, a sensor finds an intruder and, based on the conditions at that moment, the only possible route is resolved: sending an alert to the control station. However, because the sensors are moving, various situations may happen that might render the chosen route unfeasible, such as one or more sensors being outside coverage range or hardware failures. Fig. 2 (B) displays how one of the drones is left offline because the multiple signals detected exceeds its maximum processing capacity. This is driven by an event that forces sector residents to leave in mass, such as when catastrophic, astronomical, or other events occur. This shows the value of making context-based decisions because uncertainty is likely to disappear.

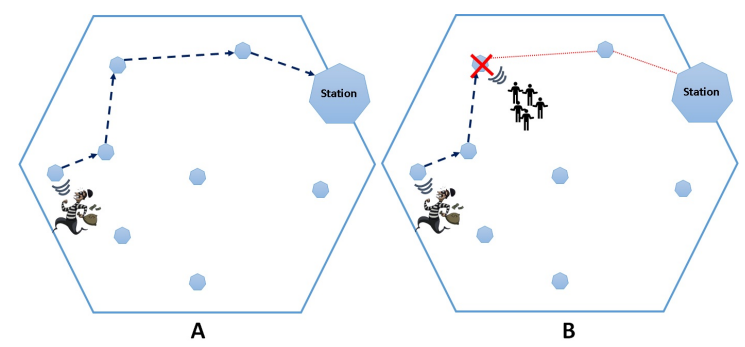

Fig. 2. IoT Scenario with Uncertainty Problems.

\subsection{Smart Spaces}

Gartner, a consulting and IT research company, believed that smart spaces were one of the ten strategic technological trends in 2019. Gartner defined smart spaces as "a physical or digital environment in which humans and technologyenabled systems interact in increasingly open, connected, coordinated and intelligent ecosystems" [6]. 
Fig. 3 denotes a scenario of a home focused on the care and wellbeing of an ill person, who requires monitoring of her activities and vital signs. Because her health may be affected by changes in light, temperature, humidity, and noise, these variables will also have to be controlled. This particular scenario depicts a house furnished with all types of sensors and portable devices used for controlling the environment and alerting a nearby hospital if needed. The idea is to provide immediate assistance by sending an ambulance. In addition, her social media are also monitored to supplement the data and, in some cases, to provide additional context to the data extracted from the sensor. Nevertheless, situations may occur in which uncertainty does not allow for a clear decision or wherein it may lead to incorrect decisions. For example, if the person decides to take a nap on the floor, it may appear that the patient has fainted or suffered an accident, depending on when the images or videos taken are seen. If a person is watching a horror movie, their pulse may be disturbed, but if the video is not able to determine what activity the person is doing, the data from portable devices may cause a false alarm. This example would show that context awareness is a significant matter when managing uncertainty in decision making.

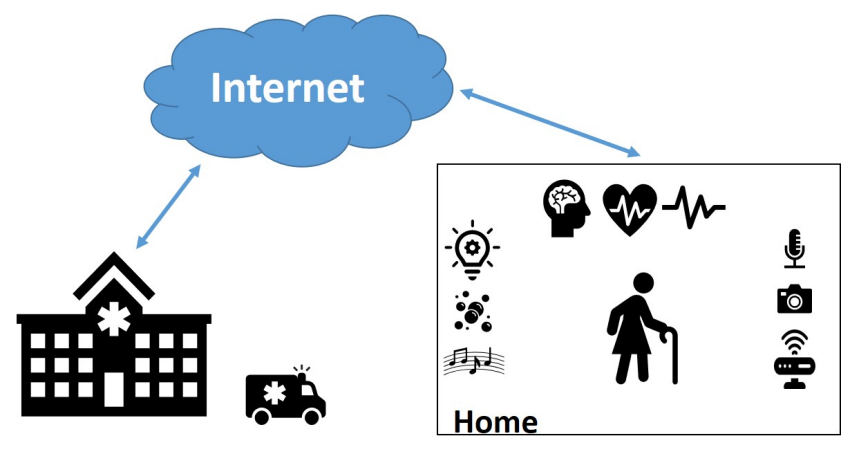

Fig. 3. Smart Space Scenario with Uncertainty

\subsection{Industry 4.0}

To examine the impact of uncertainty on Industry 4.0, let us review the scenario of a call center providing customer services for a mobile phone company. The main goal of these service companies is to provide timely attention to as many customers as feasible. Therefore, the average time of user calls is a significant factor that must be controlled because network resources are limited, whether in terms of telephone lines or bandwidth. To streamline these resources, among other technological measures adopted, the call center has a Customer Relationship Management (CRM) system to profile users and record, monitor, track, and predict events. System data are registered after receiving user consent, whether 
from social media, information from cell phones, or through other means. The information recorded in the CRM system supports processing requests received by various means such as telephone calls, social media, chats, or email in a more efficient manner. Nevertheless, if this information is not properly contextualized, the opposite effect can be created, thus actually increasing the time spent in responding to a request and using more network infrastructure resources. The following example presents a case where people uncertainty affects resource usage for the proposed scenario. Let us assume that some users have been selected to answer a loyalty survey, which has an estimated maximum timeframe. Based on these criteria, users were selected if they had stated that they were highly satisfied with the service. In addition, users had to be young, assuming that they handle new technologies better; belong to a high socioeconomic status; reside in an urban population; and have completed minimum academic levels. Nevertheless, when the call center agent places the corresponding calls, some respondents who meet all the criteria exhibit conditions or situations that significantly increase the time required to complete the survey. For example, some may have some cognitive disorder, or answer the call while performing some other activity that they continue doing concurrently as they provide their responses, such as playing video games, or some may even have some mild hearing, vision, or speech impairments. Hence, as it can be observed that Industry 4.0 features offer, in a prospective vision, deep changes and transformations, especially in terms of the workforce, social inequality, and new business model developments. The application of such technological concepts also creates new challenges faced by communications networks to support services proposed by this fourth revolution, thus proving the need for developing computational methods to solve uncertainty problems related to human beings. Therefore, several proposals have been found to solve this kind of technical problems, and they all have SDN in common. SDN are emerging communications networks that separate the control plane from the data plane to allow for interoperability, programmability, and flexibility [20] to co-exist as features. In addition, all of the studies found address the technical problems of Industry 4.0 from several SDN-compatible focuses. For example, network virtualization [23], Ethernet network metrics [34], cloud manufacturing [30], cybersecurity [14], or resource allocation and information exchange for IoT [31].

\section{Open Problems and Future Research Directions}

The increased interweaving of networks and systems with human activities creates new development possibilities within several contexts. However, this brings about several challenges in the triad assessed in this work, such as the integration of decision making under uncertainty, network resource management, and HCN. At this stage, we describe the main open research problems arising within this context, classified as presented in Fig. 4. 


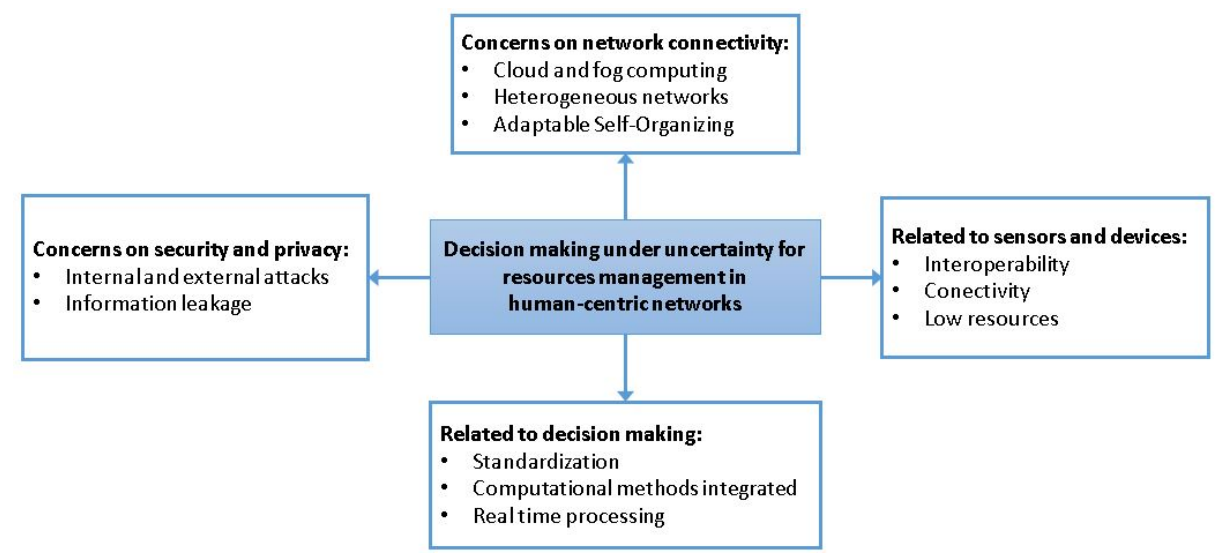

Fig. 4. Open Problem Categories

We identify, at least, the following future research directions:

- IoT massification and the deployment of the $5 \mathrm{G}$ network will cause high network densification, which brings about the need to examine new routing protocols that may support constant changes in user contexts. This means that these protocols may use context information whenever moment priorities change, employing the required resources to meet new objectives.

- The large level of ubiquity and information exchange among users and systems will facilitate security threats sustained by artificial intelligence. Therefore, there is an urgent need to set up policies and measures to protect personal information.

- HCNs can potentially generate large amounts of data due to the integration and interconnection of individuals and machines as part of their network infrastructure. The availability of data generated by people and machines brings about an opportunity to compile context awareness. In this light, a new information technology paradigm must be proposed to consider the rationality and irrationality of human behavior when managing the resources of underlying infrastructures. For instance, to suppress the data uncertainty that humans add to emerging networks, the structuralist nature of psychoanalysis can be researched to model human uncertainty.

\section{Conclusions}

In foresight, human-centric networks are destined to transform how we live our daily lives. Nevertheless, the intensive use that may be given to HCN may generate high traffic rates that will have an impact on DCN resources. However, the solution may be related to the data that generate this traffic. In this study, we presented a comprehensive review of several decision-making computational methods like MCDM, optimization algorithms, and machine learning proposed 
for dimensioning, control and deployment of future networks. These methods have been separately and jointly assessed in different fields, such as telecommunications, energy, transportation, health, business decisions, problems under uncertainty, complex systems, and for improving decision-making processes. However, various decision-making method combinations are clearly yet to be explored, especially those related to machine learning methods. In addition, we believe that a new multidisciplinary computational paradigm must be developed to address several uncertainty factors. Although it is beyond the scope of this review, information security is relevant issue due to the implications and the scope that may be feasible when processing private personal information. Therefore, we think that a strong emphasis should be placed on methods aimed at limiting potential abuses with personal data as, otherwise, the emerging concept of HCNs would lose all credibility. Finally, based on a review of emerging concepts, such as smart spaces and Industry 4.0, the lack of standardization is clearly evidenced, even in the heterogeneity of their definitions.

\section{References}

1. Almeida, A.T.d., Morais, D.C., Alencar, L.H., Clemente, T.R.N., Krym, E.M., Barboza, C.Z.: A multicriteria decision model for technology readiness assessment for energy based on PROMETHEE method with surrogate weights. In: 2014 IEEE International Conference on Industrial Engineering and Engineering Management. pp. 64-68 (2014)

2. Asadabadi, M.R.: The stratified multi-criteria decision-making method. Knowledge-Based Systems 162, 115-123 (7 2018)

3. Ashton K.: That 'Internet of Things' thing in the real world, things matter more than ideas. RFID Journal (2009)

4. Cables, E., Lamata, M., Verdegay, J.: RIM-reference ideal method in multicriteria decision making. Information Sciences 337-338, 1-10 (4 2016)

5. Cables, E.H., Lamata, M.T., Verdegay, J.L.: Ideal Reference Method with Linguistic Labels: A Comparison with LTOPSIS, pp. 115-126. Springer International Publishing, Cham (2019)

6. Cearley, D., Burke, B., Furlonger, D., Kandaswamy, R., Litan, A.: Top 10 Strategic Technology Trends for 2019. Tech. Rep. March 2019, Gartner (2019)

7. Chahuara, P., Portet, F., Vacher, M.: Context-aware decision making under uncertainty for voice-based control of smart home. Expert Systems with Applications 75, 63-79 (6 2017)

8. Chen, S.M., Cheng, S.H., Lan, T.C.: A new multicriteria decision making method based on the topsis method and similarity measures between intuitionistic fuzzy sets. In: 2016 International Conference on Machine Learning and Cybernetics (ICMLC). vol. 2, pp. 692-696. IEEE (7 2016)

9. Conti, M., Passarella, A., Das, S.K.: The Internet of People (IoP): A new wave in pervasive mobile computing. Pervasive and Mobile Computing 41, 1-27 (2017)

10. Dammak, F., Baccour, L., Ayed, A.B., Alimi, A.M.: ELECTRE method using interval-valued intuitionistic fuzzy sets and possibility theory for multi-criteria decision making problem resolution. In: IEEE International Conference on Fuzzy Systems. pp. 1-6. IEEE (7 2017) 
11. Dix, A.: Human-computer interaction, foundations and new paradigms. Journal of Visual Languages and Computing 42, 122-134 (2016)

12. Fei, X., Shah, N., Verba, N., Chao, K.M., Sanchez-Anguix, V., Lewandowski, J., James, A., Usman, Z.: CPS data streams analytics based on machine learning for Cloud and Fog Computing: A survey. Future Generation Computer Systems 90, 435-450 (1 2019)

13. Ferrara, M., Rasouli, S., Khademi, M., Salimi, M.: A robust optimization model for a decision-making problem: An application for stock market. Operations Research Perspectives 4, 136-141 (2017)

14. Fraile, F., Flores, J.L., Poler, R., Saiz, E.: Software-Defined Networking to Improve Cybersecurity in Manufacturing Oriented Interoperability Ecosystems. In: Enterprise Interoperability VIII. pp. 31-41. Cham (2019)

15. Gervasio, H., Simoes Da Silva, L.: A probabilistic decision-making approach for the sustainable assessment of infrastructures. Expert Systems with Applications 39(8), 7121-7131 (6 2012)

16. Jiang, W., Strufe, M., Schotten, H.D.: A SON decision-making framework for intelligent management in 5G mobile networks. In: 2017 3rd IEEE International Conference on Computer and Communications (ICCC). pp. 1158-1162. IEEE (12 2017)

17. Ken, S.A.F., Eldor, S.R.M., Guillemin, W.C.H., Paul, Y.W.K., Armstrong, C.D., G, I., Kim, Yong-Woon, Chartier, C.P., Praxis, Armstrong, L.D.: RFID and the inclusive model for the Internet of Things (2009)

18. Khezrimotlagh, D., Chen, Y.: Data envelopment analysis. In: International Series in Operations Research and Management Science, vol. 269, pp. 217-234. Springer, Dordrecht (2018)

19. Kochenderfer, M.J., Amato, C., Chowdhary, G., How, J.P., Reynolds, H.J.D., Thornton, J.R., Torres-Carrasquillo, P.A., Üre, N.K., Vian, J.: Decision Making Under Uncertainty: Theory and Application. MIT Lincoln Laboratory Series (2015)

20. Kreutz, D., Ramos, F.M.V., Veríssimo, P.E., Rothenberg, C.E., Azodolmolky, S., Uhlig, S.: Software-Defined Networking: A Comprehensive Survey. Proceedings of the IEEE 103(1), 14-76 (2015)

21. Kumar, G.: A multi-criteria decision making approach for recommending a product using sentiment analysis. In: 2018 12th International Conference on Research Challenges in Information Science (RCIS). pp. 1-6. IEEE (5 2018)

22. Lee, J., Bagheri, B., Kao, H.A.: A Cyber-Physical Systems architecture for Industry 4.0-based manufacturing systems. Manufacturing Letters 3, 18-23 (1 2015)

23. Ma, Y.W., Chen, Y.C., Chen, J.L.: SDN-enabled network virtualization for industry 4.0 based on IoTs and cloud computing. In: 2017 19th International Conference on Advanced Communication Technology (ICACT). pp. 199-202. IEEE (2017)

24. Morente-Molinera, J.A., Kou, G., Samuylov, K., Ureña, R., Herrera-Viedma, E.: Carrying out consensual Group Decision Making processes under social networks using sentiment analysis over comparative expressions. Knowledge-Based Systems 165, 335-345 (2019)

25. Mousavi, S.M., Gitinavard, H., Siadat, A.: A new hesitant fuzzy analytical hierarchy process method for decision-making problems under uncertainty. In: 2014 IEEE International Conference on Industrial Engineering and Engineering Management. pp. 622-626 (2014)

26. Perçin, S.: Evaluating airline service quality using a combined fuzzy decisionmaking approach. Journal of Air Transport Management 68, 48-60 (5 2018) 
27. Qin, J., Liu, X., Pedrycz, W.: An extended VIKOR method based on prospect theory for multiple attribute decision making under interval type-2 fuzzy environment. Knowledge-Based Systems 86, 116-130 (9 2015)

28. Shuwei Chen, Liu, J., Wang, H., Augusto, J.C.: An evidential reasoning based approach for decision making with partially ordered preference under uncertainty. In: 2013 International Conference on Machine Learning and Cybernetics. vol. 04, pp. 1712-1717. IEEE (7 2013)

29. Smet, Y.D.: About the computation of robust PROMETHEE II rankings: Empirical evidence. In: 2016 IEEE International Conference on Industrial Engineering and Engineering Management (IEEM). pp. 1116-1120 (2016)

30. Thames, L., Schaefer, D.: Software-defined Cloud Manufacturing for Industry 4.0. Procedia CIRP 52, 12-17 (2016)

31. Wan, J., Tang, S., Shu, Z., Li, D., Wang, S., Imran, M., Vasilakos, A.: SoftwareDefined Industrial Internet of Things in the Context of Industry 4.0. IEEE Sensors Journal 16(20), 1-1 (2016)

32. Wei, L., Yuan, Z., Yan, Y., Hou, J., Qin, T.: Evaluation of energy saving and emission reduction effect in thermal power plants based on entropy weight and PROMETHEE method. In: 2016 Chinese Control and Decision Conference (CCDC). pp. 143-146 (2016)

33. Yu, B., Cai, M., Li, Q.: A $\lambda$-rough set model and its applications with TOPSIS method to decision making. Knowledge-Based Systems 165, 420-431 (2019)

34. Zeng, P., Wang, Z., Jia, Z., Kong, L., Li, D., Jin, X.: Time-slotted software-defined Industrial Ethernet for real-time Quality of Service in Industry 4.0. Future Generation Computer Systems 99, 1-10 (2019)

35. Zhang, P., Yao, H., Qiu, C., Liu, Y.: Virtual Network Embedding Using Node Multiple Metrics Based on Simplified ELECTRE Method. IEEE Access 6, 3731437327 (2018) 